\title{
Cylindrical Carleman's formula of subharmonic functions and its application
}

\section{Lei Qiao}

School of Mathematics and Information Science, Henan University of Economics and Law, Zhengzhou 450046, China.

Communicated by R. Saadati

\begin{abstract}
Our aim in this paper is to prove the cylindrical Carleman's formula for subharmonic functions in a truncated cylinder. As an application, we prove that if the positive part of a harmonic function in a cylinder satisfies a slowly growing condition, then its negative part can also be dominated by a similar slowly growing condition, which improves some classical results about harmonic functions in a cylinder.
\end{abstract}

Keywords: Cylindrical Carleman's formula, subharmonic function, cylinder.

2010 MSC: 31B05, 31B10, 35J05, 35J10, 35J40.

(C)2018 All rights reserved.

\section{Introduction}

Let $\mathbf{R}$ be the set of all real numbers. The boundary and the closure of a set $E$ in $n$-dimensional Euclidean space $\mathbf{R}^{n}(n \geqslant 2)$ are denoted by $\partial E$ and $\bar{E}$ respectively.

Let $\Delta_{n}$ be the Laplace operator and $\Omega$ be a bounded domain in $\mathbf{R}^{n-1}$ with smooth boundary $\partial \Omega$. Consider the Dirichlet problem (see [11, p. 41])

$$
\begin{aligned}
\left(\Delta_{\mathrm{n}-1}+\lambda\right) \varphi=0 & \text { on } \Omega, \\
\varphi=0 & \text { on } \partial \Omega .
\end{aligned}
$$

We denote the least positive eigenvalue of this boundary value problem by $\lambda$ and the normalized positive eigenfunction corresponding to $\lambda$ by $\varphi$,

$$
\int_{\Omega} \varphi^{2}(X) \mathrm{d} \Omega=1
$$

where $X \in \Omega$ and $d \Omega$ is the ( $n-1)$-dimensional volume element.

The set

$$
\Omega \times \mathbf{R}=\left\{P=(X, y) \in \mathbf{R}^{n} ; X \in \Omega, y \in \mathbf{R}\right\}
$$

in $\mathbf{R}^{n}$ is simply denoted by $T_{n}(\Omega)$. We call it a cylinder (see $[5-7,12]$ ). In the following, we denote the sets

Email address: qiaocqu@163.com (Lei Qiao)

doi: $10.22436 /$ jnsa.011.08.01

Received: 2018-04-10 Revised: 2018-05-04 Accepted: 2018-05-16 
$\Omega \times I$ and $\partial \Omega \times I$ with an interval I on $\mathbf{R}$ by $T_{n}(\Omega ; I)$ and $S_{n}(\Omega ; I)$ respectively. Hence $S_{n}(\Omega ; R)$ denoted simply by $S_{n}(\Omega)$ is $\partial T_{n}(\Omega)$.

In order to make the subsequent consideration simpler, we put a rather strong assumption on $\Omega$ throughout this paper: if $n \geqslant 3$, then $\Omega$ is a $C^{2, \alpha}$-domain $(0<\alpha<1)$ in $\mathbf{R}^{n-1}$ surrounded by a finite number of mutually disjoint closed hypersurfaces (e.g. see [2, p. 88-89] for the definition of $C^{2, \alpha}$-domain).

Let $\mathcal{G}_{\Omega}(P, Q)$ be the cylindrical Green function of $T_{n}(\Omega)\left(P, Q \in T_{n}(\Omega)\right)$. Then the cylindrical Poisson kernel in $T_{n}(\Omega)$ is defined by

$$
\mathcal{P J}_{\Omega}(P, Q)=\frac{1}{c_{n}} \frac{\partial \mathcal{G}_{\Omega}(P, Q)}{\partial n_{Q}}
$$

where $\partial / \partial n_{Q}$ denotes the differentiation at $Q \in S_{n}(\Omega)$ along the inward normal into $T_{n}(\Omega)$ for any $P \in T_{n}(\Omega)$. Here, $c_{2}=2$ and $c_{n}=(n-2) w_{n}$ when $n \geqslant 3$, where $w_{n}$ is the surface area of the unit sphere in $\mathbf{R}^{n}$. It follows from our assumption on $\Omega$ that $\mathcal{P J}_{\Omega}(P, Q)$ is continuous on $S_{n}(\Omega)$ (see [2, Theorem 6.15]).

The cylindrical Poisson integral $\mathcal{P J}_{\Omega}[g](P)$ of $g$ relative to $T_{n}(\Omega)$ is defined as follows

$$
\mathcal{P J}_{\Omega}[g](P)=\int_{S_{n}(\Omega)} \mathcal{P J}_{\Omega}(P, Q) g(Q) d \sigma_{Q},
$$

where $g(Q)$ is a locally integrable function on $S_{n}(\Omega)$ and $d \sigma_{Q}$ is the surface area element on $S_{n}(\Omega)$.

Let $h(P)$ be a function on $T_{n}(\Omega)$, we use the stand notations $h^{+}=\max \{h, 0\}$ and $h^{-}=-\min \{h, 0\}$. The integral

$$
\int_{\Omega} h(P) \varphi(X) d \Omega
$$

of $h(P)$ is denoted by $N_{h}(y)$ when it exists, where $P=(X, y)$. The finite or infinite limits

$$
\lim _{y \rightarrow+\infty} e^{-\sqrt{\lambda} y} N_{h}(y) \text { and } \lim _{y \rightarrow-\infty} e^{\sqrt{\lambda} y} N_{h}(y)
$$

are denoted by $\mathscr{U}_{\sqrt{\lambda}}(\mathrm{h})$ and $\mathscr{V}_{\sqrt{\lambda}}(\mathrm{h})$ respectively, when they exist.

Recently, Qiao (see [8]) proved Carleman's formula of harmonic functions by using the second Green's formula. As for the Carleman's formulas of harmonic functions in a half-space, smooth cone and their applications, we refer the interested readers to the papers of Armitage (see [1]), Kuran (see [3]) and Ronkin (see $[4,9,10])$.

Our first aim in this paper is to prove cylindrical Carleman's formula of subharmonic functions in a truncated cylinder.

Theorem 1.1. Let $0<\mathrm{r}<\mathrm{R}<+\infty$ and define

$$
\Psi(y)=e^{\sqrt{\lambda} y}\left(\frac{1}{e^{2 \sqrt{\lambda} y}}-\frac{1}{e^{2 \sqrt{\lambda} R}}\right),
$$

where $\mathrm{r}<|\mathrm{y}|<\mathrm{R}$. If $\mathrm{u}(\mathrm{X}, \mathrm{y})$ is a subharmonic function in two domains containing $\mathrm{T}_{\mathrm{n}}(\Omega,(\mathrm{r}, \mathrm{R}))$ and $\mathrm{T}_{\mathrm{n}}(\Omega,(-\mathrm{R},-\mathrm{r}))$ respectively, then we have

$$
\int_{T_{n}(\Omega,(r, R))} \mathfrak{F}(X, y) \Delta_{n} u(X, y) d w=\frac{2 \sqrt{\lambda}}{e^{\sqrt{\lambda} R}} N_{u}(R)+\int_{S_{n}(\Omega,(r, R))} u\left(X^{\prime}, y^{\prime}\right) \Psi\left(y^{\prime}\right) \frac{\partial \varphi\left(X^{\prime}\right)}{\partial n_{X^{\prime}}} d \sigma_{Q}+d_{1}(r)+\frac{d_{2}(r)}{e^{2 \sqrt{\lambda} R}}
$$

and

$$
\begin{aligned}
\int_{T_{\mathfrak{n}}(\Omega,(-R,-r))} & \mathfrak{F}(X, y) \Delta_{\mathfrak{n}} u(X, y) d w \\
& =\frac{2 \sqrt{\lambda}}{e^{\sqrt{\lambda} R}} N_{u}(-R)+\int_{S_{n}(\Omega,(-R,-r))} u\left(X^{\prime}, y^{\prime}\right) \Psi\left(-y^{\prime}\right) \frac{\partial \varphi\left(X^{\prime}\right)}{\partial n_{X^{\prime}}} d \sigma_{Q}+d_{3}(-r)+\frac{d_{4}(-r)}{e^{2 \sqrt{\lambda} R},}
\end{aligned}
$$


respectively, where $\mathrm{d} w$ denotes the elements of the Euclidean volume in $\mathbf{R}^{\mathrm{n}}$,

$$
\begin{aligned}
\mathfrak{F}(X, y) & =\Psi(y) \varphi(X) \\
d_{1}(r) & =\int_{\Omega}-\frac{\varphi(X)}{e^{\sqrt{\lambda} r}}\left(\sqrt{\lambda} u(X, r)+\frac{\partial u(X, r)}{\partial n}\right) d \Omega, \\
d_{2}(r) & =\int_{\Omega} e^{\sqrt{\lambda} r} \varphi(X)\left(\frac{\partial u(X, r)}{\partial n}-\sqrt{\lambda} u(X, r)\right) d \Omega, \\
d_{3}(-r) & =\int_{\Omega}-\frac{\varphi(X)}{e^{\sqrt{\lambda} r}}\left(\sqrt{\lambda} u(X,-r)+\frac{\partial u(X,-r)}{\partial n}\right) d \Omega,
\end{aligned}
$$

and

$$
d_{4}(-r)=\int_{\Omega} e^{\sqrt{\lambda} r} \varphi(X)\left(\frac{\partial u(X,-r)}{\partial n}-\sqrt{\lambda} u(X,-r)\right) d \Omega
$$

As an application of Theorem 1.1, we give the integral representation of harmonic functions on $T_{n}(\Omega)$. To do this, we denote $\mathscr{A}_{\Omega}$ the class of $f(X, y)\left((X, y) \in \mathrm{T}_{\mathrm{n}}(\Omega)\right)$ satisfying

$$
\int_{-\infty}^{+\infty} e^{-\sqrt{\lambda}|y|}\left(\int_{\Omega}|f(P)|^{p} \varphi(X) d \Omega\right) d y<+\infty
$$

and $\mathscr{B}_{\Omega}$ the class of $\mathrm{g}(\mathrm{Q})\left(\mathrm{Q}=\left(\mathrm{X}^{\prime}, \mathrm{y}^{\prime}\right) \in \mathrm{S}_{\mathrm{n}}(\Omega)\right)$ such that

$$
\int_{-\infty}^{+\infty} e^{-\sqrt{\lambda}\left|y^{\prime}\right|}\left(\int_{\partial \Omega}|g(Q)|^{p} \frac{\partial \varphi\left(X^{\prime}\right)}{\partial n_{X^{\prime}}} d \sigma_{X^{\prime}}\right) d y^{\prime}<+\infty,
$$

where $1 \leqslant p<\infty$. We denote by $\mathscr{C}_{\Omega}$ the class of all continuous $h(X, y)\left((X, y) \in \overline{T_{n}(\Omega)}\right)$ harmonic on $\mathrm{T}_{\mathrm{n}}(\Omega)$ with $\mathrm{h}^{+}(\mathrm{X}, \mathrm{y}) \in \mathscr{A}_{\Omega}\left((\mathrm{X}, \mathrm{y}) \in \mathrm{T}_{\mathrm{n}}(\Omega)\right)$ and $\mathrm{h}^{+}(\mathrm{Q}) \in \mathscr{B}_{\Omega}\left(\mathrm{Q}=\left(\mathrm{X}^{\prime}, \mathrm{y}^{\prime}\right) \in \mathrm{S}_{\mathrm{n}}(\Omega)\right)$.

As an application of Theorem 1.1, we have the following result with weaker integral boundary conditions, which is due to Qiao (see [8]) in the case $p=1$.

Theorem 1.2. If $\mathrm{h} \in \mathscr{C}_{\Omega}$, then $\mathrm{h} \in \mathscr{B}_{\Omega}$.

\section{Lemmas}

Lemma 2.1 (see [12, Theroem 6]). Let $g(Q)\left(Q=\left(X^{\prime}, y^{\prime}\right)\right)$ be a continuous function on $S_{n}(\Omega)$ satisfying

$$
\int_{-\infty}^{+\infty} e^{-\sqrt{\lambda}\left|y^{\prime}\right|}\left(\int_{\partial \Omega}|g(Q)| d \sigma_{X^{\prime}}\right) d y^{\prime}<+\infty,
$$

where $d \sigma_{X^{\prime}}$ is the surface area element of $\partial \Omega$ at $X^{\prime} \in \partial \Omega$. Then the cylindrical Poisson integral $\mathcal{P J}_{\Omega}[\mathrm{g}](\mathrm{P})$ is a solution of the Dirichlet problem on $\mathrm{T}_{\mathrm{n}}(\Omega)$ with $\mathrm{g}$ and satisfies

$$
\mathscr{U}_{\sqrt{\lambda}}\left(\mathcal{P J}_{\Omega}[g]\right)=0
$$

and

$$
\mathscr{V}_{\sqrt{\lambda}}\left(\mathcal{P J}_{\Omega}[\mathrm{g}]\right)=0
$$

Lemma 2.2 (see [5, Corollary 3]). Let $\mathrm{h}(\mathrm{P})(\geqslant 0)$ be a harmonic function on $\mathrm{T}_{\mathrm{n}}(\Omega)$ vanishing continuously on $S_{n}(\Omega)$, then $h(P)$ admits the following representation

$$
h(P)=\left(\mathscr{U}_{\sqrt{\lambda}}(h) e^{\sqrt{\lambda} y}+\mathscr{V}_{\sqrt{\lambda}}(h) e^{-\sqrt{\lambda} y}\right) \varphi(X)
$$

for any $\mathrm{P}=(\mathrm{X}, \mathrm{y}) \in \mathrm{T}_{\mathrm{n}}(\Omega)$. 


\section{Proof of Theorems}

\subsection{Proof of Theorem 1.1}

To prove (1.1). It is achieved by a similar argument in [8, Theorem 1.4]. From the definition of $\mathfrak{F}(X, y)$, we know that $\mathfrak{F}(X, y)$ is harmonic on $T_{n}(\Omega)$ and vanishes continuously on $S_{n}(\Omega)$.

By applying the second Green's formula to $u(X, y)$ and $\mathfrak{F}(X, y)$ on $T_{n}(\Omega ;(r, R))$, we see that

$$
\begin{aligned}
I(X, y) & =: \int_{T_{n}(\Omega ;(r, R))}\left(u(X, y) \frac{\partial \mathfrak{F}(X, y)}{\partial n}-\mathfrak{F}(X, y) \frac{\partial u(X, y)}{\partial n}\right) d w \\
& =\int_{T_{n}(\Omega ;(r, R))} \mathfrak{F}(X, y) \Delta_{n} u(X, y) d w,
\end{aligned}
$$

where $\partial / \partial n$ denotes the differentiation along the inward normal into $T_{n}(\Omega ;(r, R))$. Put

$$
I(X, y)=I_{1}(X, y)+I_{2}(X, y)+I_{3}(X, y),
$$

where

and

$$
\begin{aligned}
& I_{1}(X, y)=\int_{\Omega}\left(\left.u(X, R) \frac{\partial \mathfrak{F}(X, y)}{\partial n}\right|_{y=R}-\left.\mathfrak{F}(X, R) \frac{\partial u(X, y)}{\partial n}\right|_{y=R}\right) d \Omega \\
& I_{2}(X, y)=\int_{\Omega}\left(\left.u(X, r) \frac{\partial \mathfrak{F}(X, y)}{\partial n}\right|_{y=r}-\left.\mathfrak{F}(X, r) \frac{\partial u(X, y)}{\partial n}\right|_{y=r}\right) d \Omega
\end{aligned}
$$

$$
I_{3}(X, y)=\int_{\left.S_{n}(\Omega ;(r, R))\right)}\left(u\left(X^{\prime}, y^{\prime}\right) \frac{\partial \mathfrak{F}\left(X^{\prime}, y^{\prime}\right)}{\partial n}-\mathfrak{F}\left(X^{\prime}, y^{\prime}\right) \frac{\partial u\left(X^{\prime}, y^{\prime}\right)}{\partial n}\right) d \sigma_{Q}
$$

It is easy to see that

$$
\begin{gathered}
\mathfrak{F}(X, R)=0,\left.\quad \frac{\partial \mathfrak{F}(X, y)}{\partial n}\right|_{y=R}=\frac{2 \sqrt{\lambda}}{e^{\sqrt{\lambda} R}} \varphi(X), \\
\left.\frac{\partial \mathfrak{F}(X, y)}{\partial n}\right|_{y=r}=-\sqrt{\lambda} e^{\sqrt{\lambda} r}\left(\frac{1}{e^{2 \sqrt{\lambda} r}}+\frac{1}{e^{2 \sqrt{\lambda} R}}\right) \varphi(X), \\
\mathfrak{F}(X, y)=0 \text { and } \frac{\partial \mathfrak{F}\left(X^{\prime}, y^{\prime}\right)}{\partial n}=\Psi\left(y^{\prime}\right) \frac{\partial \varphi\left(X^{\prime}\right)}{\partial n_{X^{\prime}}} .
\end{gathered}
$$

Thus (1.1) follows from (3.1), (3.2), (3.3), (3.4) and (3.5). We omit the proof of (1.2), since it can be proved similarly.

\subsection{Proof of Theorem 1.2}

In order to make the proof simpler we prove only the case $p=1$, since the proof of the case $p>1$ is similar by using Hölder inequality. We apply (1.1) and (1.2) with $R>r=1$ to $h=h^{+}-h^{-}$in $T_{n}(\Omega ;(1, R))$ and $T_{n}(\Omega ;(-R,-1))$ respectively, and then obtain that

$$
\begin{aligned}
m_{+}(R)+\int_{S_{n}(\Omega,(1, R))} & h^{+}(Q) \Psi\left(y^{\prime}\right) \frac{\partial \varphi\left(X^{\prime}\right)}{\partial n_{X^{\prime}}} d \sigma_{Q}+d_{1}(1)+\frac{d_{2}(1)}{e^{2 \sqrt{\lambda} R}} \\
& =m_{-}(R)+\int_{S_{n}(\Omega,(1, R))} h^{-}(Q) \Psi\left(y^{\prime}\right) \frac{\partial \varphi\left(X^{\prime}\right)}{\partial n_{X^{\prime}}} d \sigma_{Q}
\end{aligned}
$$


and

$$
\begin{aligned}
m_{+}(-R)+\int_{S_{n}(\Omega,(-R,-1))} & h^{+}(Q) \Psi\left(-y^{\prime}\right) \frac{\partial \varphi\left(X^{\prime}\right)}{\partial n_{X^{\prime}}} d \sigma_{Q}+d_{3}(-1)+\frac{d_{4}(-1)}{e^{2 \sqrt{\lambda} R}} \\
& =m_{-}(-R)+\int_{S_{n}(\Omega,(-R,-1))} h^{-}(Q) \Psi\left(-y^{\prime}\right) \frac{\partial \varphi\left(X^{\prime}\right)}{\partial n_{X^{\prime}}} d \sigma_{Q},
\end{aligned}
$$

respectively, where $Q=\left(X^{\prime}, y^{\prime}\right)$,

$$
m_{ \pm}(R)=\frac{2 \sqrt{\lambda}}{e^{\sqrt{\lambda} R}} N_{h^{ \pm}}(R) \quad \text { and } \quad m_{ \pm}(-R)=\frac{2 \sqrt{\lambda}}{e^{\sqrt{\lambda} R}} N_{h^{ \pm}}(-R) .
$$

Without loss of generality we can assume $R>2$, we have from (3.6) and (3.7)

$$
\begin{aligned}
m_{-}(R)+ & \left(1-\frac{1}{e^{\sqrt{\lambda} R}}\right) \int_{1}^{\frac{R}{2}} e^{-\sqrt{\lambda} y^{\prime}}\left(\int_{\partial \Omega} h^{-}(Q) \frac{\partial \varphi\left(X^{\prime}\right)}{\partial n_{X^{\prime}}} d \sigma_{X^{\prime}}\right) d y^{\prime} \\
& \leqslant m_{-}(R)+\int_{S_{n}(\Omega,(1, R))} h^{-}(Q) \Psi\left(y^{\prime}\right) \frac{\partial \varphi\left(X^{\prime}\right)}{\partial n_{X^{\prime}}} d \sigma_{Q} \\
& \leqslant m_{+}(R)+\int_{1}^{R} e^{-\sqrt{\lambda} y^{\prime}}\left(\int_{\partial \Omega} h^{+}(Q) \frac{\partial \varphi\left(X^{\prime}\right)}{\partial n_{X^{\prime}}} d \sigma_{X^{\prime}}\right) d y^{\prime}+\left|d_{1}(1)\right|+\left|d_{2}(1)\right|
\end{aligned}
$$

and

$$
\begin{aligned}
m_{-}(-R)+ & \left(1-\frac{1}{e^{\sqrt{\lambda} R}}\right) \int_{-\frac{R}{2}}^{-1} e^{\sqrt{\lambda} y^{\prime}}\left(\int_{\partial \Omega} h^{-}(Q) \frac{\partial \varphi\left(X^{\prime}\right)}{\partial n_{X^{\prime}}} d \sigma_{X^{\prime}}\right) d y^{\prime} \\
& \leqslant m_{-}(-R)+\int_{S_{n}(\Omega,(-R,-1))} h^{-}(Q) \Psi\left(-y^{\prime}\right) \frac{\partial \varphi\left(X^{\prime}\right)}{\partial n_{X^{\prime}}} d \sigma_{Q} \\
& \leqslant m_{+}(-R)+\int_{-R}^{-1} e^{\sqrt{\lambda} y^{\prime}}\left(\int_{\partial \Omega} h^{+}(Q) \frac{\partial \varphi\left(X^{\prime}\right)}{\partial n_{X^{\prime}}} d \sigma_{X^{\prime}}\right) d y^{\prime}+\left|d_{3}(-1)\right|+\left|d_{4}(-1)\right|,
\end{aligned}
$$

respectively.

Since $h \in \mathscr{C}_{\Omega}$, we obtain from (1.3)

$$
\int_{1}^{+\infty} m_{+}(R) d R=2 \sqrt{\lambda} \int_{1}^{+\infty} e^{-\sqrt{\lambda} y} N_{h^{+}}(y) d y<+\infty
$$

and

$$
\int_{1}^{+\infty} m_{+}(-R) d R=2 \sqrt{\lambda} \int_{-\infty}^{-1} e^{\sqrt{\lambda} y} N_{h^{+}}(y) d y<+\infty
$$

which give that

$$
\lim _{R \rightarrow+\infty} m_{+}(R)<+\infty
$$

and

$$
\lim _{R \rightarrow+\infty} m_{+}(-R)<+\infty
$$

respectively.

Combining (1.3), (3.8), (3.9), (3.10) and (3.11), we can conclude that

$$
\int^{+\infty} e^{-\sqrt{\lambda} y^{\prime}}\left(\int_{\partial \Omega} h^{-}(Q) \frac{\partial \varphi\left(X^{\prime}\right)}{\partial n_{X^{\prime}}} d \sigma_{X^{\prime}}\right) d y^{\prime}<+\infty
$$


and

$$
\int_{-\infty} e^{\sqrt{\lambda} y^{\prime}}\left(\int_{\partial \Omega} h^{-}(Q) \frac{\partial \varphi\left(X^{\prime}\right)}{\partial n_{X^{\prime}}} d \sigma_{X^{\prime}}\right) d y^{\prime}<+\infty
$$

which give that

$$
\int_{-\infty}^{+\infty} e^{-\sqrt{\lambda}\left|y^{\prime}\right|}\left(\int_{\partial \Omega} h^{-}(Q) \frac{\partial \varphi\left(X^{\prime}\right)}{\partial n_{X^{\prime}}} d \sigma_{X^{\prime}}\right) d y^{\prime}<+\infty
$$

Hence Theorem 1.2 is proved from $|h|=h^{+}-h^{-}$.

\section{Acknowledgment}

The author thanks the referees for their valuable comments. This work was supported by the National Natural Science Foundation of China (Grant Nos. 11301140, U1304102), the Foundation for University Young Key Teacher Program of Henan Province (Grant No. 2017GGJS085) and Young Talents Fund of HUEL.

\section{References}

[1] D. H. Armitage, A Nevanlinna theorem for superharmonic functions in half-spaces, with applications, J. London Math. Soc., 23 (1981), 137-157. 1

[2] D. Gilbarg, N. S. Trudinger, Elliptic partial differential equations of second order. Grundlehren der Mathematischen Wissenschaften, Springer-Verlag, Berlin-New York, (1977). 1

[3] Ü. Kuran, Harmonic majorizations in half-balls and half-spaces, Proc. London Math. Soc., 21 (1970), 614-636. 1

[4] B. Y. Levin, Entire and subharmonic functions, Adv. Soviet Math., Amer. Math. Soc., Providence, RI, (1992). 1

[5] I. Miyamoto, Harmonic functions in a cylinder which vanish on the boundary, Japan. J. Math., 22 (1996), 241-255. 1, 2.2

[6] I. Miyamoto, H. Yoshida, Harmonic functions in a cylinder with normal derivatives vanishing on the boundary, Ann. Polon. Math., 74 (2000), 229-235.

[7] I. Miyamoto, H. Yoshida, On a covering property of minimally thin sets at infinity in a cylinder, Math. Montisnigri, 20/21 (2007/08), 35-54. 1

[8] L. Qiao, Asymptotic behavior of Poisson integrals in a cylinder and its application to the representation of harmonic functions, Bull. Sci. Math., 144 (2018), 39-54. 1, 1, 3.1

[9] A. Y. Rashkovskǐ̌, L. I. Ronkin, Subharmonic functions of finite order in a cone. III. Functions of completely regular growth, J. Math. Sci., 77 (1995), 2929-2940. 1

[10] L. I. Ronkin, Functions of completely regular growth, Kluwer Academic Publishers Group, Dordrecht, (1992). 1

[11] G. V. Rozenblyum, M. Z. Solomyak, M. A. Shubin, Spectral theory of differential operators, Itogi Nauki i Tekhniki, Akad. Nauk SSSR, Vsesoyuz. Inst. Nauchn. i Tekhn. Inform., Moscow, (1989). 1

[12] H. Yoshida, Harmonic majorization of a subharmonic function on a cone or on a cylinder, Pacific J. Math., 148 (1991), 369-395. 1, 2.1 